\title{
ANATOMIA COMPARATIVA DE HOJA Y TALLO EN LOS REPRESENTANTES DE CESTREAE G. DON (SOLANACEAE) DE ARGENTINA
}

\section{COMPARATIVE ANATOMY OF LEAF AND STEM IN SPECIES OF CESTREAE G. DON (SOLANACEAE) FROM ARGENTINA}

\author{
Iris J. Liscovsky \& María T. Cosa
}

Instituto Multidisciplinario de Biología Vegetal - CONICET, Facultad de Ciencias Exactas Físicas y Naturales, Universidad Nacional de Córdoba. Av. Vélez Sárfield 299- (5000) Córdoba. Argentina.

iliscovsky@imbiv.unc.edu.ar

\section{RESUMEN}

\begin{abstract}
En este trabajo se compara la anatomía de la hoja y del tallo de las 13 especies nativas y cultivadas de Cestrum L. y en Sessea vestioides (Schltdl.) Hunz., de la tribu Cestreae G. Don (Solanaceae) que crecen en Argentina. Se utilizó material de herbario y conservado en FAA. Los caracteres que permitieron una mejor delimitación a nivel específico en la hoja son: la arquitectura de la vena secundaria y de la venación última marginal, los tipos de estomas y tricomas, las características de la cutícula, la presencia de fibras en relación al floema de la vena media y la ocurrencia y abundancia de esclereidas en mesófilo y vena media. En el tallo las características diagnósticas son: la presencia y abundancia de esclereidas en la epidermis, córtex y médula; la presencia de fibras en relación al floema y el origen de la peridermis. Sessea vestioides, la única especie de este género citada para Argentina, no presentó caracteres diferenciales con respecto a Cestrum en el análisis de los órganos vegetativos.
\end{abstract}

Palabras Claves: Arquitectura, Cestrum, morfología, órganos vegetativos, Sessea.

\section{ABSTRACT}

This article compares leaf and stem anatomy of the 13 native and cultivated species of Cestrum L. and Sessea vestioides (Schltdl.) Hunz., of the tribe Cestreae G. Don (Solanaceae) from Argentina. Herbarium and FAA-preserved material was employed. The leaf characters which best distinguished among species were: secondary vein architecture, marginal ultimate venation, stomata and hairs, cuticle characteristics, presence of fibres adjacent to external and internal phloem in the middle vein, sclereid presence and abundance in mesophyll and middle vein. In stems the best diagnostic characters were: sclereid presence and abundance in epidermis, cortex and pith, fibre presence adjacent to phloem, and the periderm origin. We did not identify any vegetative characters that clearly distinguished Sessea vestioides, the only species of the genus in Argentina, from Cestrum.

Keywords: Architecture, Cestrum, morphology, Sessea, vegetative organs.

\section{INTRODUCCION}

La tribu Cestreae G. Don, incluida en la subfamilia Cestroideae Schltdl., comprende tres géneros de plantas leñosas, generalmente arbustivas: Cestrum L., Sessea Ruiz et Pav. y Vestia Willd. Los dos primeros tienen representantes nativos en Argentina (Hunziker 2001), mientras que Vestia, género monotípico, crece en Chile.
Cestrum es el género más numeroso de la subfamilia, contando con unas 150-200 especies (Hunziker 2001). En Argentina se encuentran 11 representantes nativos (Romanutti \& Hunziker 1998): C. albotomentosum Dammer ex Francey, $C$. amictum Schltdl., C. euanthes Schltdl., $C$. guaraniticum Chodat et Hassl., C. kunthii Francey, C. laevigatum $\mathrm{Schltdl}$., C. lorentzianum Griseb., $C$. parqui L'Hér., $C$. reflexum Sendtn., $C$. 
sendtnerianum Mart. y C. strigillatum Ruiz et Pav. Además se cultivan dos especies exóticas (Ratera 1964): C. elegans (Brongn.) Schltdl. y C. nocturnum L. Este género tiene importancia económica ya que comprende especies medicinales (Marzocca 1997) y otras tóxicas para el ganado (Gallo 1987; González Stuart 1989; Matthei 1995). C. parqui es la de mayor distribución en Argentina, transmite el virus del tabaco a los cultivos (Ramallo 1967), es tóxica para el ganado (Yagueddú et al. 2000) y tiene un probado poder insecticida (Rafael et al. 2000).

Sessea comprende unas 25 especies algunas de las cuales también han sido mencionadas como tóxicas para el ganado, tal es el caso de S. brasiliensis Toledo (Andrade et al. 1970). En Argentina crece únicamente S. vestioides (Schltdl.) Hunz. (Romanutti \& Hunziker 1998), y en lugares muy restringidos.

A pesar del gran número de especies que comprende la tribu Cestreae y su amplia distribución en Latinoamérica, los estudios de arquitectura, morfología o anatomía son escasos. Este vacío mantiene la dificultad taxonómica que representa Cestrum y que resalta Nee (2001), ya que las especies son separadas en general por datos exomorfológicos, que muchas veces no resultan ser suficientes. Sessea tampoco ha sido objeto de muchos estudios, por lo que las controversias taxonómicas, acerca de unificar o mantener separados estos géneros íntimamente relacionados, permanecen vigentes. Scolnik (1954) considera que entre los géneros Cestrum y Sessea no existen diferencias morfológicas notables, siendo el tipo de fruto, la forma y presencia de alas en la semilla, las características principales que permiten delimitar ambos géneros.

Este trabajo describe y compara la arquitectura, morfología y anatomía de hoja, y la anatomía del tallo de las especies nativas y cultivadas de Cestreae de Argentina, a fin de posibilitar su determinación taxonómica cuando se carece de flores o frutos, como también facilitar la identificación de sus fragmentos en caso de intoxicaciones (Yagueddú et al. 2000).

\section{MATERIALES Y METODOS}

Se utilizó material fresco, conservado en FAA y de herbario. La mayoría de los ejemplares se encuentran depositados en el Museo Botánico de Córdoba (CORD), indicándose la sigla de herbario sólo en aquellos que no están en CORD. Además de ejemplares de
Argentina se han analizado otros de Bolivia, Brasil y Paraguay.

Cestrum albotomentosum Dammer ex Francey. ARGENTINA. Prov. Catamarca: Dpto. Andalgalá, Río Potrero, 22-II-2003, Barboza et al. 624. Prov. Salta: Dpto. Orán, Aguas Blancas, 12-V-1989, Novara et al. 8918. BOLIVIA. Dpto. Santa Cruz: Prov. Andrés Ibañez, 30-I-1987, Nee y Coimbra 33869.

Cestrum amictum Schltdl. ARGENTINA. Prov. Misiones: Dpto. Cainguás, San Antonio, Colonia Manuel Belgrano, 20-X-1972, Eskuche 4-89. BRASIL. Edo. Paraná: Mun. Tibagí, 3-VIII-1953, Hatschbach 3484. Edo. Santa Catarina: Itajai, 29I-1948, Reitz 2039.

Cestrum elegans (Brongn.) Schltdl. ARGENTINA. Prov. Buenos Aires: Distrito Federal, Villa Ortúzar, Fac. de Agronomía (UBA), 25-XI-1942, A. T. Hunziker 2872, “cult.”; Jardín Botánico Lucién Hauman, Fac. de Agronomía (UBA), 29-X-2002, Tortosa s/n, "cult.".

Cestrum euanthes Schltdl. ARGENTINA. Prov. Buenos Aires: Delta del Paraná, 17-X-1942, A. T. Hunziker 3211. Prov. Corrientes: Dpto. Monte Caseros, Ruta 122 y Arroyo Timboy, 10-IX-1979, Schinini et al. 18291. Prov. Entre Ríos: Dpto. Paraná, Arroyo Martínez, 13-X-1944, Burkart 15126; Dpto. Uruguay, Concepción del Uruguay, 25-IX-1961, Burkart 22715; Dpto. Colón, Parque Nacional El Palmar, 13-XII-1975, Burkart et al. 27408.

Cestrum guaraniticum Chodat et Hassl. ARGENTINA. Prov. Entre Ríos: Dpto. La Paz, Isla Curuzú Chalí, 9-11-IV-1968, Burkart et al. 27069. Prov. Misiones: Dpto. Capital, en playas del Río Paraná, 7-XII-2002, Barboza et al. 405.

Cestrum kunthii Francey. ARGENTINA. Prov. Tucumán: Dpto. Tafi, cerca de Taficillo, 14-IX1952, Sleumer 3051; Las Azucenas, 11-XI-1952, A. T .Hunziker 10087; Arroyo Las Azucenas, 7IV-2003, Barboza et al. 776.

Cestrum laevigatum Schltdl. ARGENTINA. Prov. Corrientes: Dpto. San Martín, Laguna Iberá, 31X-1971, Krapovickas et al. 20257. Prov. Misiones: Dpto. Posadas, Gurupá, 12-V-1949, Bertoni 2888 (LIL).

Cestrum lorentzianum Griseb. ARGENTINA. Prov. Catamarca: Dpto. Andalgalá, Río Chacras, 7-III1998, Barboza et al. 157; Río Potreros, 7-III-1998, Barboza et al. 158. Prov. Córdoba: Dpto. Colón, 
camino a Salsipuedes, 2-XII-2001, Cosa 333, 24III-2002, Cosa 337; Dique La Quebrada, 19-X2002, Liscovsky 29; 27-XII-2002, Liscovsky 35. Prov. Jujuy: Dpto. Tumbaya, Purmamarca, 19-X2001, Cocucci 1394bis.

Cestrum nocturnum L. ARGENTINA. Prov. Catamarca: Dpto. Andalgalá, Río Potreros, 3-V1996, A. T. Hunziker 25596. Prov. Córdoba: Dpto. Capital, Córdoba, Fac. de Ciencias Exactas Físicas y Naturales, UNC, 29-III-2002, Ariza s/n, “cult.”.

Cestrum parqui L'Hér. ARGENTINA. Prov. Córdoba: Dpto. Capital, Córdoba, 28-XI-2001, Cosa 329; Dpto. Colón, La Calera, 3-X-2001, Liscovsky 6; camino a Salsipuedes, 2-XI-2001, Cosa 332; Dique La Quebrada, 19-X-2002, Liscovsky 28, 27-XII-2002, Liscovsky 34. Prov. Entre Ríos: Dpto. Paraná, Paraná, 17-III-02, Cosa 335. Prov. La Pampa: Dpto. Capital, Santa Rosa, 24-X-2001, Liscovsky 11.

Cestrum reflexum Sendtn. ARGENTINA. Prov. de Salta: Dpto. Orán, Vado Hondo, 16-III-1969, Legname y Cuezo 6122C (LIL); camino a Quebrada de Arrasayan, 15-III-1969, Legname y Cuezo 6123C (LIL); Río las Moras, 7-V-1969, Legname y Cuezo 6179 (LIL). BOLIVIA. Dpto. La Paz: Coroico, 26/30-VIII-1947, Scolnik y Luti 490 “cult.". Dpto. Santa Cruz Prov. Andrés Ibáñez, Santa Cruz, 14-VII-1987, Nee 35162.

Cestrum sendtnerianum Mart. ARGENTINA. Prov. Corrientes: Dpto. Beron Astrada, Laguna Toroy, 21-X-1949, Schwarz 8384 (LIL). Prov. Misiones: Dpto. General Manuel Belgrano, Puerto Aguirre, XI-1940, Jiménez 8859; Dpto. Cainguás, Puerto Francés, 1-VIII-1949, Schwarz 7974. BOLIVIA. Dpto. Santa Cruz: Prov. Ichilo, La Arboleda, 30I-1988, Nee 36131, San Javier, 21-V-1991, Nee 40493.

Cestrum strigillatum Ruiz et Pav. ARGENTINA. Prov. Catamarca: Prov. Fitogeográfica de las Yungas, Selva Montana, XII-2001, Lucero s/n. Prov. Misiones: Dpto. Apóstoles, Apóstoles, 11-I-2002, Chiarini 539; Dpto. Candelaria, Santa Ana, 8-XII2002, Barboza et al. 454. Prov. Tucumán: Dpto. Capital, Tucumán, Instituto Miguel Lillo, 6-VIII2002, Barboza y Matesevach 333bis „ "cult.”.

Sessea vestioides (Schltdl.) Hunz. ARGENTINA. Prov. Corrientes: Dpto. Santo Tomé, Timbó, 16XI-1980, Ahumada y Schinini, 4089 (CTES). PARAGUAY. Cordillerita, VIII-1932, Rojas 6062; Acahay, Chodat 44, sin otros datos.
El material de herbario se hidrató previamente con hidróxido de sodio al $5 \%$ bajo el punto de ebullición, se enjuagó con agua destilada y se aclaró con hipoclorito de sodio al 50\%. Para los estudios de arquitectura foliar el material fue diafanizado siguiendo la técnica de Dizzeo de Strittmater (D’Ambrogio 1986) y se lo tiñó con safranina diluida en alcohol $70 \%$. Se utilizó la técnica de "peeling" para la observación de la epidermis en vista superficial y se tiñó con la doble coloración azul astral-safranina.

La estructura interna de la hoja y del tallo se estudió en preparados temporarios de cortes transversales, coloreados con azul astral-safranina o en preparados permanentes. Para estos últimos el material fijado en FAA se deshidrató en serie de alcohol etílico y xilol y se lo incluyó en histoplast. Los cortes microtómicos de 10 y $15 \mu \mathrm{m}$ de espesor se colorearon con hematoxilina activada, safranina y verde permanente (Conn et al. 1960). Las observaciones se efectuaron con microscopio óptico Kyowa.

Se utilizó la clasificación de Hickey (1974) para arquitectura foliar, y en la clasificación de estomas basada en la ontogenia se siguió a FrynsClaessens \& Van Cotthen (1973). Se calculó en todas las especies la frecuencia estomática en término de la relación entre estomas y células epidérmicas propiamente dichas (Índice Estomático), haciendo de esta medida un valor comparable independientemente del tamaño foliar y de las condiciones ambientales (Stace 1965).

\section{RESULTADOS}

\section{HOJA}

Arquitectura foliar: En todas las especies analizadas hay una vena media principal (venación pinnada) y de acuerdo al comportamiento de las venas secundarias se encontraron dos tipos de venación. 1) broquidódroma, cuando las venas secundarias no terminan en el margen y se unen en arcos prominentes, y 2) semicraspedódroma, cuando las venas secundarias se ramifican próximas al margen, una de las ramas termina en el margen y la otra se une a la vena secundaria supradyacente. El primer tipo incluye a la mayoría de las especies de Cestrum, y el segundo tipo a C. albotomentosum, $C$. euanthes, $C$. laevigatum, $C$. lorentzianum, $C$. parqui, $C$. reflexum y $S$. vestioides. 
La venación última marginal es generalmente fimbriada, con una vena fimbrial o colectora en el margen, y menos frecuentemente ojalada, formando ojales en el margen en $C$. amictum, $C$. euanthes y Sessea vestioides. (Tabla I, Figs. 8-11).

EPIDERMIS EN VISTA SUPERFICIAl: La epidermis presenta células con paredes onduladas, de mayor tamaño en el epifilo que en el hipofilo y distribuidas al azar (Fig. 12). La cutícula es generalmente lisa; se observan estriaciones en ambas caras de las hojas de $C$. lorentzianum, $C$. parqui y $S$. vestiodes o sólo en el hipofilo en $C$. kunthii. En el epifilo la ornamentación es menos notable que en el hipofilo y está restringida a ambos lados de la vena media, o rodeando estomas o tricomas (Tabla I).

La clasificación de los tricomas responde a la siguiente descripción:

1. Eglandulares simples (Fig. 1): multicelulares y uniseriados, de forma cónica o filiforme. La cutícula es generalmente lisa, a veces con estriaciones.

2. Eglandular ramificado: multicelulares y uniseriados, con una o varias ramas. Se distinguen dos subtipos de acuerdo al grado de complejidad:

2.1. Ramificado de primer orden (Fig. 2): las ramas se forman al prolongarse en sentido oblicuo o transversal las células de la única hilera del tricoma.

2.2. Ramificado de segundo orden (Fig. 3): las ramas constan de más de una célula e incluso pueden volver a ramificarse.

3. Glandulares: con pie de una a cinco células uniseriado, se distinguen dos subtipos de acuerdo a la cabezuela.

3.1. Glandulares con cabezuela unicelular (Fig. 4): cabezuela de forma ovoidea o redondeada.

3.2. Glandulares con cabezuela bicelular (Figs. 57): las células de la cabezuela se disponen en sentido longitudinal respecto al eje del tricoma.

Los tricomas tanto eglandulares como glandulares predominan en el hipofilo, excepto en $C$. amictum donde son abundantes en el epifilo. Los tipos hallados según las especies se señalan en la Tabla I.

Las hojas son generalmente hipostomáticas, ocasionalmente hay estomas en el epifilo de Cestrum kunthii, $C$. sendtnerianum, $C$. nocturnum y $C$. lorentzianum. Sólo $C$. parqui presenta hojas anfistomáticas, pero siempre son más abundantes los estomas en la cara abaxial (Tabla I). El índice estomático promedio para el hipofilo es de $19 \pm 0,7$ (varía entre 13,7 en $C$. amictum y 26,4 en $C$. elegans).

En todas las especies analizadas predominan los estomas sin células auxiliares o aperígenos (Fig. 13), y en menor proporción con una célula auxiliar paralela o perpendicular a las oclusivas, tipos hemiparamesoperígenos y hemidiamesoperígenos, respectivamente; o con dos auxiliares paralelas a las oclusivas, o tres de diferente tamaño rodeando a las oclusivas, tipos paramesoperígeno y anisomesoperígeno, respectivamente (Figs. 14 y 15 y Tabla I). Por último, se han observado estomas atípicos, contiguos (Fig. 13) o con una o dos células oclusivas abortivas.

Estructura interna: La lámina en sección transversal presenta en ambas caras la epidermis unistrata, el mesofilo es dorsiventral y consta de una capa de parénquima en empalizada (excepcionalmente algunas células sufren división transversal) y un número variable de capas en el parénquima esponjoso (de 3 a 7). Los haces vasculares están rodeados por una vaina parenquimática (Fig. 17). En C. amictum se observan escasas esclereidas en el mesofilo.

La vena media en general es prominente en ambas caras. En transcorte se observa colénquima subepidérmico y por dentro parénquima, en cambio en Sessea vestioides es poco prominente en la cara adaxial y no presenta colénquima. El haz vascular tiene forma de arco o "U". En relación al floema interno o externo, o a ambos, pueden presentarse fibras (Tabla I, Fig.16); en Cestrum parqui y $C$. lorentzianum, la presencia y abundancia de fibras es variable en diferentes poblaciones, aunque en la mayoría de los casos están presentes. Solamente dos especies presentan esclereidas a este nivel; en $C$. laevigatum son abundantes rodeando el floema en la zona abaxial de la hoja y en $C$. amictum son escasas en el parénquima que rodea al haz. Todos los haces vasculares de la lámina se encuentran rodeados por una vaina parenquimática, que en algunas especies es muy notable.

El pecíolo generalmente es ensanchado en la zona proximal. En sección transversal presenta la epidermis unistrata, un tejido parenquimático subepidérmico con escasas esclereidas, caso de $C$. amictum y C. reflexum. Las características del haz vascular son similares a las señaladas en la lámina, 


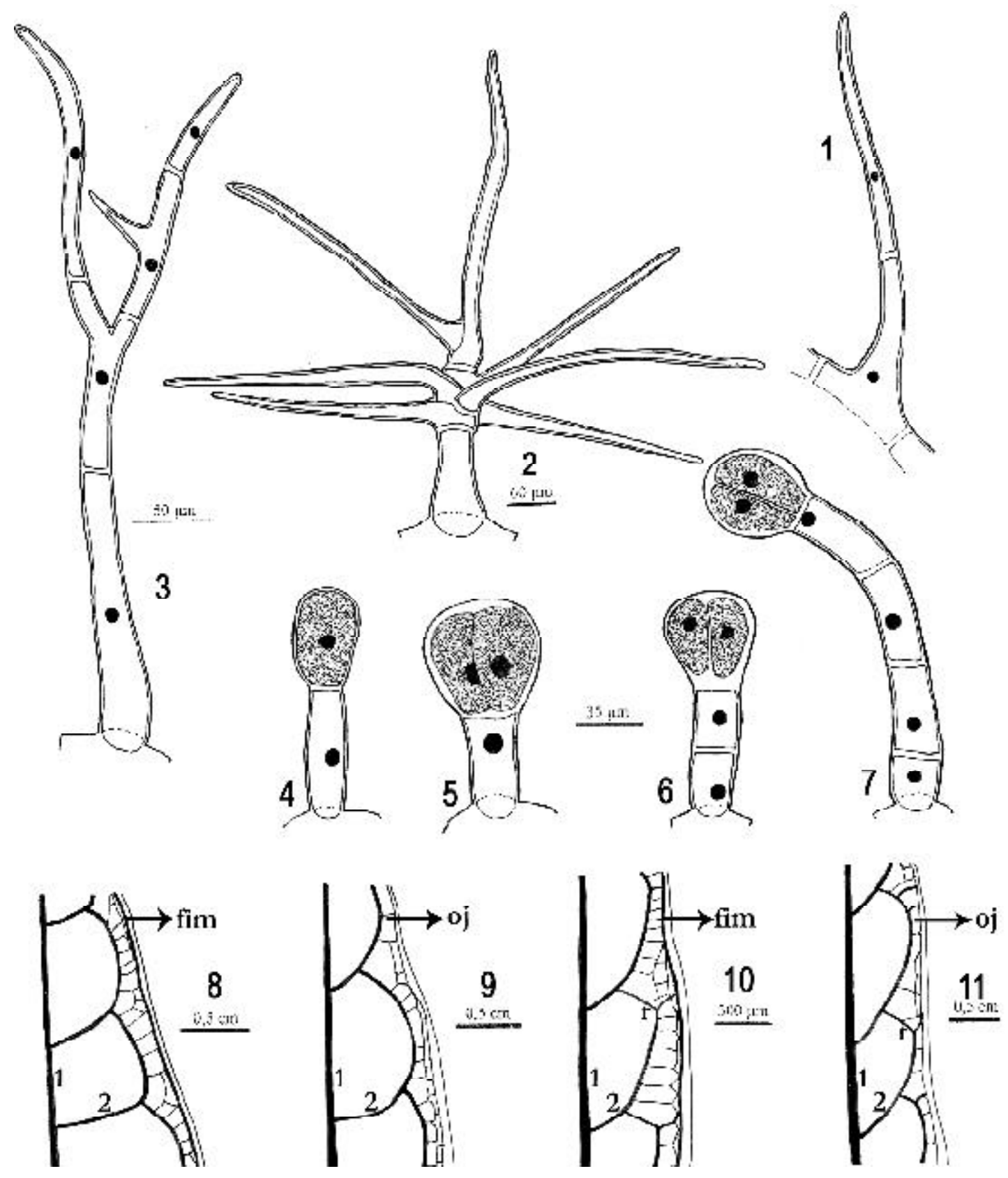

Figuras 1-7. Tricomas. Figs. 1 y 3: C. lorentzianum, Barboza et al. 158; Fig. 1: eglandular simple; Fig. 3: eglandular ramificado de segundo orden; Fig. 2: C. strigillatum, Lucero s/n, eglandular ramificado de primer orden. Fig. 4: C. amictum, Reit 2039, glandular con cabezuela unicelular. Figs. 5-7: glandular con cabezuela bicelular; Fig. 5: $C$. nocturnum, cult.; Fig. 6: C. parqui, Cosa 329; Fig. 7: C. lorentzianum, Cocucci 1394bis.

Figuras 8-11: Arquitectura foliar. Fig. 8: estructura broquidódroma con margen fimbriado, C. elegans, A. T. Hunziker 2872; Fig. 9: estructura broquidódroma con margen ojalado, C. amictum, Gratachbach 3484; Fig. 10: estructura semicraspedódroma con margen fimbriado, C. parqui, Liscovsky 6; Fig. 11: estructura semicraspedódroma con margen ojalado, S. vestioides, Chodat 44. Referencias: 1: vena primaria; 2: vena secundaria; fim: vena fimbrial; oj: ojales; r: ramificación de la vena secundaria. Escalas: la escala de $50 \mu \mathrm{m}$ corresponde a 1 y 3 ; la escala de $35 \mu \mathrm{m}$ corresponde a $4-7$. 
TABLA I. Arquitectura, epidermis y estructura interna de la hoja de Cestreae en Argentina. Abreviaturas: bi: cabezuela bicelular; fe: floema externo; fi: floema interno; $\mathrm{r}^{\circ}$ : ramificado de primer orden; $\mathrm{r}^{\circ}$ : ramificado de segundo orden; s: simple; uni: cabezuela unicelular.

\begin{tabular}{|c|c|c|c|c|c|}
\hline & $\begin{array}{l}\text { Veración } \\
\text { secomdaria }\end{array}$ & $\begin{array}{l}\text { Venación } \\
\text { úlima } \\
\text { margiral }\end{array}$ & Cutioula & $\begin{array}{l}\text { Posición } \\
\text { estomas }\end{array}$ & sperigros \\
\hline C dBatomentosidn & semirraspedódrouma & finzbriads & lisa & hịostromática & 1701 \\
\hline C amictum & broquidótroma & ojalada & lisa & hịostromática & 53,7 \\
\hline C elegons & broquidotrom & fimbriada & lisa & hịostromática & 190,5 \\
\hline Ceusuties & semiraspedódroma & ojalada & lisa & hịostrarática & 1723 \\
\hline C. gerarariticum & broquidótrom & finmbriada & lisa & hịostrmática & 1849 \\
\hline C notin & broquidótroma & finbriada & estriada & hịostromática & 1429 \\
\hline $\begin{array}{l}\text { C. bavigatom } \\
\text { C borentrionum }\end{array}$ & semi- sspedódroma & $\begin{array}{l}\text { finbriada } \\
\text { finmbriada }\end{array}$ & $\underset{\text { estriada }}{\text { lisa }}$ & $\begin{array}{l}\text { híostrmática } \\
\text { hipostrmática }\end{array}$ & $\begin{array}{l}199 f \\
1489\end{array}$ \\
\hline A notionom & broquidódrom & fimbriada & lisa & hịostromática & 62,4 \\
\hline $\begin{array}{l}\text { C. propia } \\
\text { C. reflexis }\end{array}$ & 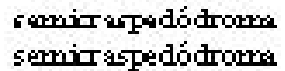 & $\begin{array}{l}\text { finkriads } \\
\text { fimbriads }\end{array}$ & $\begin{array}{l}\text { striids } \\
\text { lisa }\end{array}$ & $\begin{array}{l}\text { uf itamátics } \\
\text { hịostrórica }\end{array}$ & $\begin{array}{l}00,3 \\
1553\end{array}$ \\
\hline C sencinerionum & broquidótroma & fimbriada & lisa & híostromática & $161 p$ \\
\hline C triginathom & broquidótroma & finbriada & lisa & hipostromática & 1848 \\
\hline S vesticiales & semitaspedódroma & ojalada & estriada & hịostorrática & 1497 \\
\hline
\end{tabular}

\begin{tabular}{|c|c|c|c|c|c|c|c|c|}
\hline & \multicolumn{4}{|c|}{ Estomas por mm2 } & \multicolumn{2}{|c|}{ Tricomas } & \multicolumn{2}{|c|}{ Wena Media } \\
\hline & hamiquar- & remiatis- & sriso- & Fari- & & & & \\
\hline & & esoperigen & & & egkndulares & glandulares & fîras & esclereidas \\
\hline C abatawertcoum & 1,1 & - & - & - & $s, \mathrm{I}^{\circ}$ & - & - & - \\
\hline C awictum & 136 & 68 & 32,5 & 1,5 & 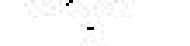 & bi, uni & fi,fe & escasas \\
\hline C elegans & 249 & 283 & - & - & $s, \mathrm{r}^{\circ}$ & bi & - & - \\
\hline Celoruthes & $28 p$ & 189 & - & - & - & bi & - & - \\
\hline C gupraniticus & 272 & 113 & - & - & $s$ & bi & fi,fe & - \\
\hline C. bostin & 83 & $5 \overline{3}$ & - & - & $s, \mathrm{r}^{*}$ & bi & fi,fe & - \\
\hline C knevigctom & 29,5 & 20,4 & - & - & 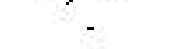 & bi & fi,fe & aburidarte \\
\hline C istertsionuon & 17,4 & 68 & - & - & $s, \mathrm{r}^{*}$ & bi & $\mathrm{fe}$ & - \\
\hline C noturnom & 272 & 57 & - & - & 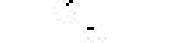 & $\mathrm{bi}$ & fi,fe & - \\
\hline C. parqan & 95 & 42 & - & - & $s, \mathrm{r}^{\circ}$ & bi & fe & - \\
\hline C reflexum & 215 & 238 & raro & - & - & bi & fi,fe & escasas \\
\hline C sencinerionum & 57 & 91 & - & - & $s$ & & fi,fe & - \\
\hline C triginamow & 24,4 & $13 p$ & - & - & $s, \mathrm{rl}^{*}$ & uni & fi,fe & - \\
\hline S vestiaides & 91 & 68 & - & - & $s$ & bi, uni & fe & - \\
\hline
\end{tabular}

también pueden hallarse fibras o esclereidas en relación al floema, generalmente son más abundantes en la zona proximal y pueden disminuir o desaparecer en la zona distal del pecíolo.

\section{TALLO}

Los tallos son circulares en sección transversal. En la estructura primaria se observa una epidermis unistrata, la cutícula es lisa o presenta ornamentaciones a manera de estrías. El córtex consta de una a dos capas subepidérmicas de colénquima, y entre cinco y ocho capas de parénquima con células redondeadas que se agrandan y posteriormente se alargan en sentido transversal con el desarrollo del órgano. El sistema vascular forma una sifonostela anfifloica y la médula es parenquimática.

En estados más avanzados del desarrollo la epidermis tiene generalmente algunas o todas las células lignificadas o transformadas en esclereidas (Fig. 19). El estrato subepidérmico se divide para dar origen a la peridermis excepto en $C$. strigillatum y $S$. vestioides donde este tejido lo origina la epidermis (Fig. 19). 


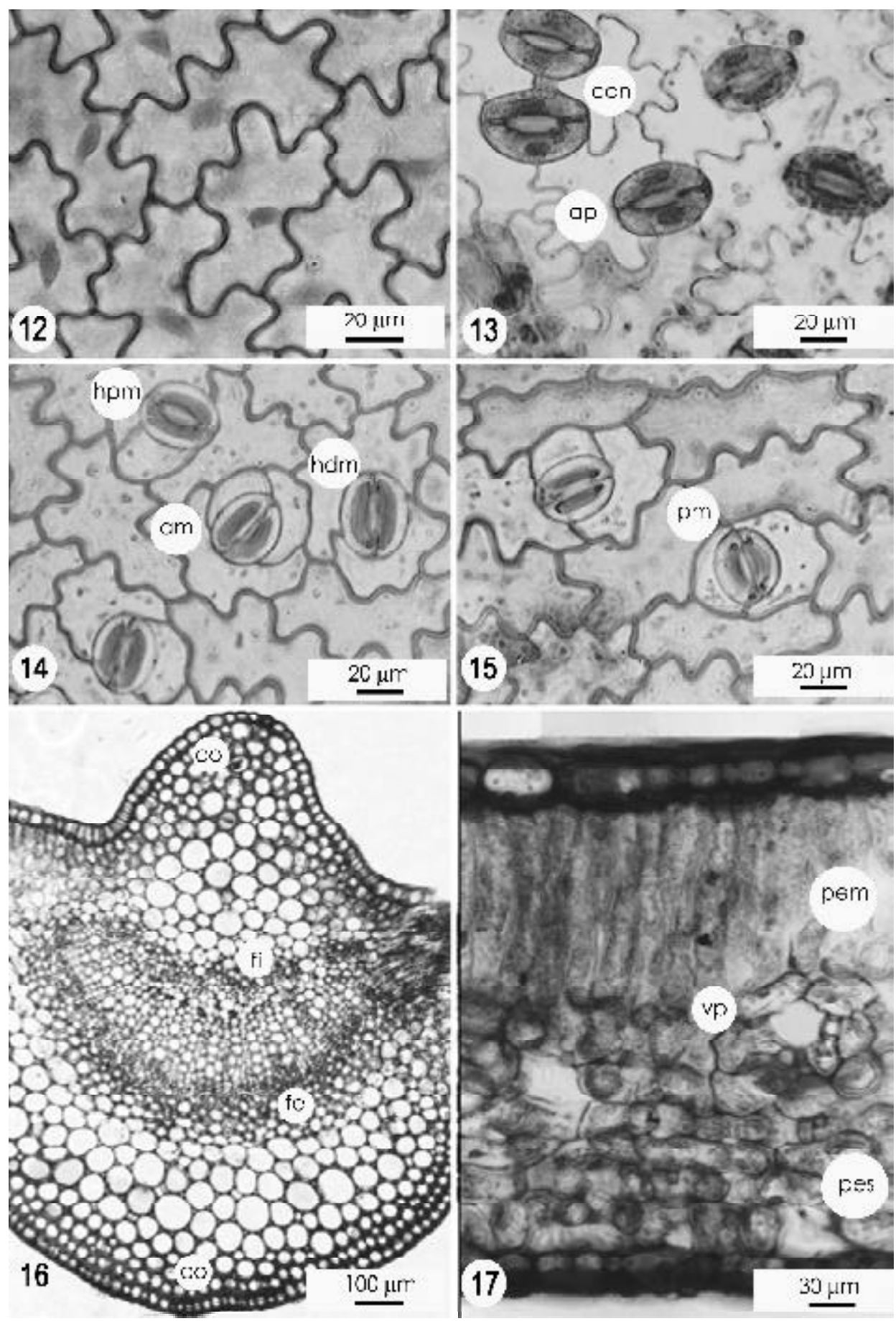

Figuras 12-17. Hoja. Fig. 12: epifilo, C. nocturnum, A. T. Hunziker 25596; Figs. 13-15: hipofilo: Fig. 13: $C$. nocturnum, A. T. Hunziker 25596; Figs. 14 y 15: C. amictum, Gratachbach 3484. Figuras 16 y 17: Anatomía foliar, C. guaraniticum, Barboza et al. 405: Fig. 16: vena media de lámina; Fig. 17: mesofilo. Referencias: am: anisomesoperígeno, ap: aperígeno, co: colénquima, con: contiguos, fe: floema externo, fi: floema interno, hdm: hemidiamesoperígeno, hpm: hemiparamesoperígeno, pem: parénquima en empalizada, pes: parénquima esponjoso, pm: paramesógeno, vp: vaina parenquimática. 
Gayana Bot. 61(2), 2005

TABLA II. Estructura interna de tallo de Cestreae en Argentina. Esclereidas en epidermis, córtex y médula: abundantes $=>50 \%$ de las células son esclereidas; escasas $=<50 \%$ de las células son esclereidas.

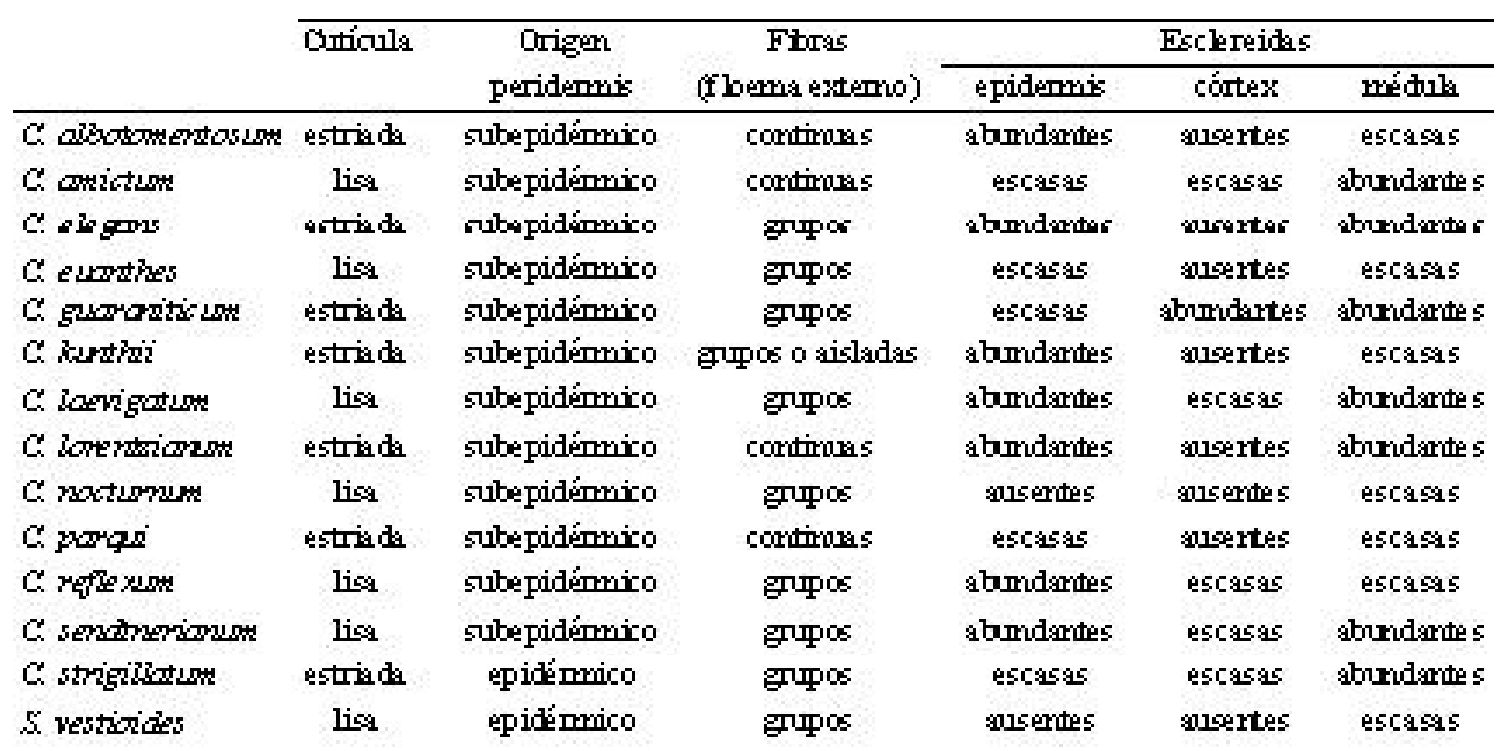

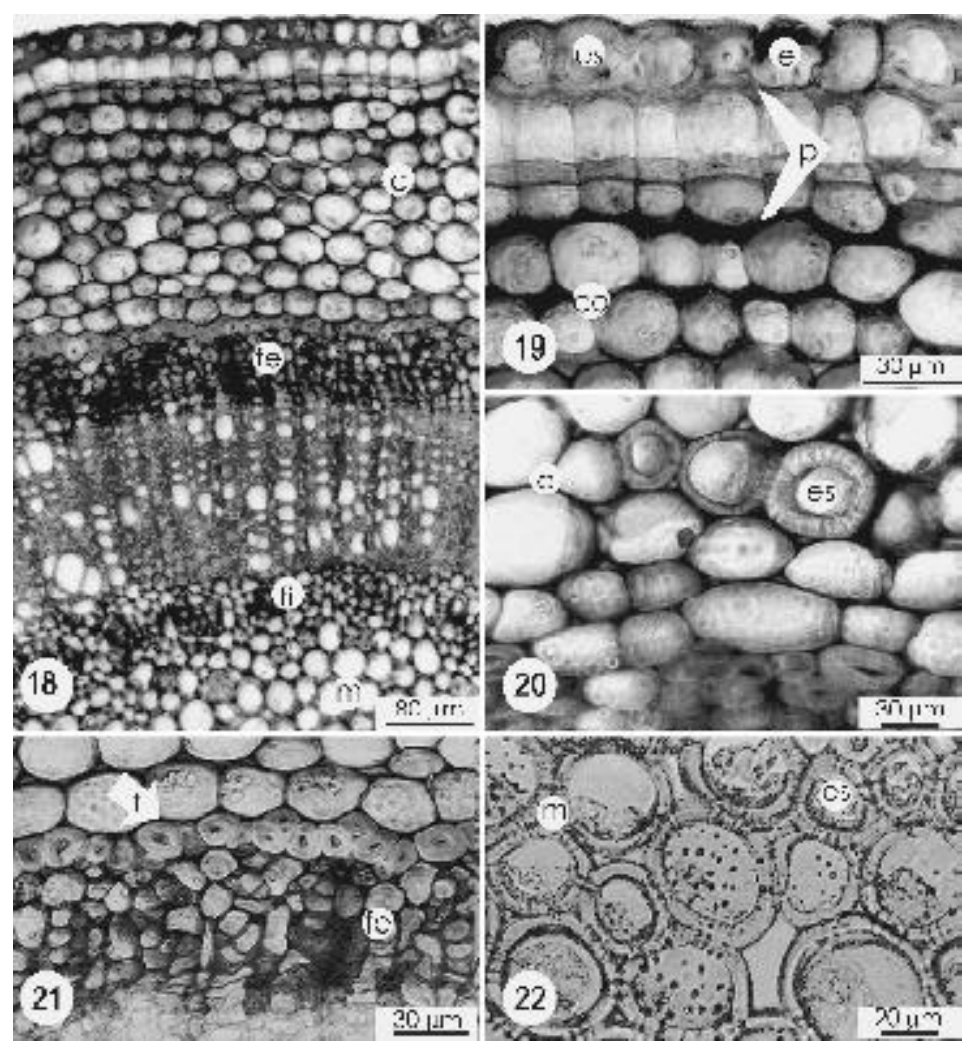

Figuras 18-22: Tallo. Figs. 18, 19 y 21, C. elegans Tortosa s/n; Fig. 18 vista general de sector de tallo secundario; Fig. 19: detalle del origen subepidérmico de la peridermis; Fig. 21: fibras en relación al floema externo; Fig. 20: esclereidas presentes en córtex, $C$. kunthii Barboza et al. 776; Fig. 22: esclereidas en médula, C. lorentzianum Cosa 337. Referencias: c: corteza, e: epidermis, es: esclereidas, f: fibras, fe: floema externo, fi: floema interno, m: médula, p: peridermis. 
En algunas especies se diferencian esclereidas en el córtex (Fig. 20) y en la médula (Fig. 22) y generalmente hay fibras en relación al floema interno y/o externo (Figs. 18 y 21), las que varían en cuanto a su distribución (en bandas continuas, grupos o aisladas) y tamaño. En $C$. kunthii, C. laevigatum y C. strigillatum se observaron además esclereidas incluidas en el floema externo (Tabla II).

Clave dicotómica

Se considera que caracteres documentados arriba permitirían la identificación a nivel específico. Se resumen los resultados en una clave dicotómica:

1. Venación secundaria broquidódroma.

2. Venación marginal ojalada C. amictum

2. Venación marginal fimbriada

3. Con cutícula estriada . C. kunthii

3. Cutícula lisa

4. Sólo tricomas eglandulares, o glandulares

5. Tricomas eglandulares solamente

C. sendtnerianum

5. Tricomas glandulares solamente ...C. nocturnum

4. Con tricomas eglandulares y glandulares

6. Con tricomas eglandulares ramificados y glandulares de cabezuela unicelular. Peridermis de origen epidérmico .C. strigillatum

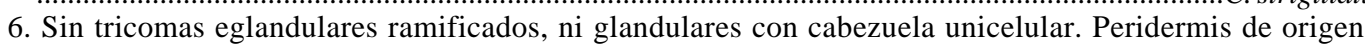
subepidérmico

7. Presencia de fibras en relación al floema interno y externo en vena media de lamina y pecíolo ..C. guaraniticum

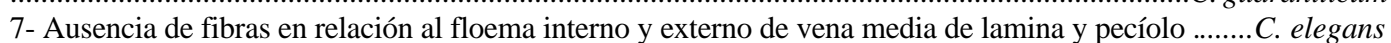
1. Venación secundaria semicraspedódroma

8. Venación marginal ojalada.

9. Con cutícula lisa y tricomas glandulares. Peridermis de origen subepidérmico C. euanthes

9. Con cutícula estriada y tricomas glandulares y eglandulares. Peridermis de origen epidérmico ........S. vestioides 8. Venación marginal fimbriada.

10. Hojas anfistomáticas

10. Hojas hipostomáticas C. parqui

11. Con tricomas eglandulares simples y sin esclereidas en córtex caulinar.

12. Sin tricomas glandulares. Sin esclereidas en epidermis de tallo .....

12. Con tricomas glandulares. Con esclereidas en epidermis de tallo

11. Sin tricomas eglandulares simples y escasas esclereidas en córtex caulinar.

13. Esclereidas abundantes rodeando la vena media de lámina y en médula del tallo

13. Esclereidas escasas rodeando la vena media de lámina y en médula del tallo C. lorentzianum ..C. laevigatum C. reflexum

\section{DISCUSION}

En los estudios foliares, en general se observó un alto grado de similitud entre las características de las especies de Argentina y de especies analizadas por otros autores. Respecto a la venación se presentaron los tipos broquidódromo y semicraspedódromo y la venación marginal es frecuentemente fimbriada, pero también se observa el tipo ojalado, en coincidencia con lo citado en estudios previos (Inamdar \& Murthy 1978; Benítez \& Ferrarotto 1997a, 1997b, 2000).

En las especies aquí descriptas, además de los estomas aperígenos o anomocíticos señalados por Jáuregui et al. (1998) y Benítez \& Ferrarotto (1997a, 1997b, 2000) se observaron con menor frecuencia estomas con una y dos células auxiliares mesógenas paralelas o perpendiculares a las oclusivas, equivalentes a los tipos paracíticos y anisocíticos de la clasificación de Metcalfe \& Chalk (1950). La presencia de varios tipos de estomas en una misma hoja se considera un carácter común en Solanaceae (Karatela \& Gill 1986). Los estomas atípicos se valoraron asumiendo la postura que son característicos de las especies y no producto de inducción ecológica ni fisiológica (Ahmad 1964). 
Los tricomas glandulares con cabezuela multicelular y con las células perpendiculares al eje citados para C. humboldtii Francey (Jáuregui et al.2000), no fueron observados en las especies aquí analizadas.

En cuanto a la anatomía foliar, no se observó una vaina completamente esclerenquimática, como se cita para $C$. salicifoliumJacq., C. megalophyllum Dunal y C. olivaceum Francey, ni una vaina parenquimática notable en los haces del mesofilo, como es el caso de C. microcalyx Francey, C. pariense Steyerm. y C. scandens Vahl, tampoco la ocurrencia de dos capas de parénquima en empalizada en la lámina, como sucede en $C$. potaliifolium Dunal., C. lindenii Dunal, C. megalophyllum y C. petiolare Kunth (Jáuregui et al. 1998, 2000). Por último nuestras observaciones coinciden con lo señalado por Hunziker (2001) en cuanto a la carencia de areniscas cristalinas en especies de Cestrum.

Las características del tallo primario en general coinciden con las descriptas por Ratera (1964) para especies de Cestrum; aunque a diferencia de este autor se observaron esclereidas en $C$. strigillatum, en epidermis, corteza y médula, y en la médula de $C$. nocturnum. El origen de la peridermis resultó ser predominantemente subepidérmico, originada a partir de la capa colenquimática subepidérmica, sólo en $C$. strigillatum y $S$. vestioides el origen es epidérmico.

Tal como describe Nee (2001), Cestrum resultó ser un género con características uniformes. Además, no presentó caracteres diferenciales en el estudio de órganos vegetativos en comparación con $S$. vestioides, lo que estaría de acuerdo a lo esperado por las observaciones de Scolnik (1954). En coincidencia con nuestros resultados, las características foliares descriptas para S. corymbiflora (Lindoford 1997) tampoco presentan diferencias con las observadas en Cestrum. Sin embargo, debido a que las disimilitudes entre estos géneros a nivel exomorfológico están dadas por el fruto y semilla, se espera que futuros estudios morfo-anatómicos basados en órganos reproductivos aporten datos para la delimitación genérica, mientras que los datos de órganos vegetativos permitan una mejor delimitación específica.

\section{AGRADECIMIENTOS}

A la Dra. Gloria Barboza y sus colaboradores y a los Herbarios CORD, LIL y CTES por facilitar material vegetal y contribuir con la determinación. A la Dra. Nilda Dottori por la lectura crítica del manuscrito. A la Secretaría de Ciencia y Técnica de la Universidad Nacional de Córdoba por el financiamiento económico de los estudios y al Consejo Nacional de Investigaciones Científicas y Técnicas por el respaldo al investigador.

\section{BIBLIOGRAFIA}

Ahmad, K. J. 1964. On stomatal abnormalities in Solanaceae. Science \& Culture 30: 349-351.

Andrade, S. O., L. Retz, M. C. F. Linardi \&A. A. Aguilar. 1970. Studies on Sessea brasiliensis Toledo II. Isolation of a hepatotoxic component from the fruit. Anais Academia Brasileira de Ciências. 42 (Suplem.): 223-233.

Benítez, C. \& M. Ferrarotto. 1997a. Arquitectura foliar en Cestrum (Solanaceae-Cestreae) de Venezuela II. Memoria Sociedad Ciencias Naturales La Salle 57: 25-35.

Benítez, C. \& M. Ferrarotto. 1997b. Arquitectura foliar en tres especies de Cestrum (Solanaceae-Cestreae) de Venezuela. Anales de Botánica Agrícola 4: 5-9.

Benítez, C. \& M. Ferrarotto. 2000. Arquitectura foliar en seis especies de Cestrum (Solanaceae-Cestreae) presentes en selvas nubladas de Venezuela. Acta Científica Venezolana 51: 10-17.

ConN, H. J., M.A. Darrow \& V. M. Emmel. 1960. Staining procedures. Editorial Williams Wilkins Co., Baltimore. $289 \mathrm{pp}$.

D’Ambrogio de Argüeso, A. 1986. Manual de Técnicas en Histología Vegetal. Editorial Hemisferio Sur, Buenos Aires. $83 \mathrm{pp}$

Fryns-Claessens, E. \& W. Van Cotthem. 1973. A new classification of the ontogenetic types of stomata. Botanical Review 39: 71-138.

Gallo, G. G. 1987. Plantas tóxicas para el ganado en el Cono Sur de América. Editorial Hemisferio Sur, Buenos Aires. 213 pp.

Gonzélez Stuart, A. 1989. Plantas tóxicas para el ganado. Editorial Limusa, México. 273 pp.

HiCKEY, L. J. 1974. Clasificación de la arquitectura de las hojas de dicotiledóneas. Boletín de la Sociedad Argentina de Botánica XVI: 1-26.

Hunziker, A. 2001. The genera of Solanaceae. A. R. G. Gantner Verlag K. G. Liechtenstein, Königstein. $500 \mathrm{pp}$

InAmdar, J. A. \& G. S. R. Murthy. 1978. Leaf Architecture in some Solanaceae. Flora 167: 265-272.

Jáuregui, D., N. R. De Ríos \& C. Benítez. 1998. Estudios anatómicos foliares en Solanaceae de Venezuela. 
Anatomía comparativa de hoja y tallo en Cestreae: Liscovsky, I. \& M.T. CosA

IV. Anatomía foliar de las especies: Cestrum bigibbosum Pitter, C. microcalyx Francey, $C$. potaliifolium Dunal, Cestrum racemosum Ruiz et Pavon y C. salicifolium Jacq., miembros de la tribu Cestreae-Solanaceae. Pittieria 28: 17-30.

Jáuregui, D., N. Rodríguez \& C. Benítez. 2000. Estudios anatómicos foliares en Solanaceae de Venezuela. V. Anatomía foliar de once especies de Cestrum L. Acta Científica Venezolana 51: 6977.

Karatela, Y. \& L. S. Gill. 1986. Observation on the developmental studies of stomatal differentiation in the epidermis of Solanaceae. Feddes Repertorium 97: 303-311.

LindFord, H. 1997. Wood and leaf anatomy in Sessea corymbiflora from an ecological perspective. IAWA Journal 18: 157-168.

M Aтthei, J. O. 1995. Manual de las malezas que crecen en Chile. Alfabeta Impresores, Santiago. 545 pp.

MarzocCA, A. 1997. Vademécum de malezas medicinales de la Argentina indígenas y exóticas. Orientación Gráfica Editora, Buenos Aires. 363 pp.

Metcalfe, C. R. \& L. Chalk. 1950. Anatomy of the Dicotyledons II. Solanaceae Clarendon Press. Oxford. $1500 \mathrm{pp}$.

NeE, M. 2001. An overview of Cestrum. Solanaceae V: Advances in Taxonomy and utilization. pp. 109136. Eds. R.G. van den Berg, G.W.M. Barendse, G..M. van der Weerden, C. Mariani. Published by Nijmegen University Press. Holanda.

Rafael, B., G. MaregGiani, A. Fraschina \& G. Bilotti. 2000.
Determinación de la actividad insecticida de Solanum sisymbriifolium, Cestrum parqui, Chenopodium album sobre adultos de Tribolium castaneum (Coleoptera, Tenebrinidae). Revista Facultad de Agronomía 20: 373-377.

Ramallo, J. C. 1967. Cestrum parqui L'Hérit, Solanum argentinum Bitter et Lillo yNicotiana longiflora Cav., malezas portadora del mosaico común del tabaco (Nicotiana virus 1 Smith) en Tucumán. Revista Agronómica Noroeste Argentino 6: 103111.

RATERA, E. L. 1964. Estudios histológicos en tallos jóvenes de Cestrum argentinos. Revista del Instituto Municipal de Botánica 2: 79-87.

Romanutti, A. \& A. Hunziker. 1998. Cestreae. En: Flora Fanerogámica Argentina, fasc. 55, (ed. A. T. Hunziker) pp. 1-14. Programa Proflora, CONICET, Córdoba.

Scolnik, R. 1954. Las especies de Cestrum de la Argentina, Chile y Uruguay. pp. 27-29. Dirección General de Publicidad de la Universidad Nacional de Córdoba. Córdoba, Argentina.

Stace, C. A. 1965. Cuticular studies as an aid to plant taxonomy. Bulletin of British Museum (Natural History) Botany 4: 62.

Yagueddú, C., M. S. Cid, T. López \& M. A. B Rizuela. 2000. Exactitud y precisión en la cuantificación por microanálisis de Cestrum parqui L. Hérit. En el contenido digestivo de ovinos en pastoreo. Revista Argentina de Producción Animal 20: $67-75$. 